Arnaldo Dubin

Fabio D. Masevicius

\section{Improving acid-base evaluation: the proper use of the old tools}

Accepted: 24 August 2009

Published online: 24 October 2009

(C) Copyright jointly hold by Springer and ESICM 2009

An author's reply to this comment is available at:

doi:10.1007/s00134-009-1682-2.

Sir: We read with interest the publication from Dr. Boniatti and colleagues [1]. Nevertheless, we believe some points deserve a careful discussion.

The authors stated: "The main result of this study is that it demonstrates the greater sensitivity of physicochemical evaluation in identifying acid-base disorders in critically ill patients." These results, however, might have been anticipated because of the study's methodological limitations. Unfortunately, one cannot show that the strong ion difference [SID] has a greater sensitivity than the base excess [BE] when different sensitivity thresholds are used for each parameter. The authors considered that the normal $\mathrm{BE}$ is -5 to $5 \mathrm{mmol} / \mathrm{l}$ and the normal effective [SID] is 38 to $42 \mathrm{mmol} / \mathrm{l}$. Consequently, the diagnosis of metabolic acidosis required a decrease in the base buffer [BB] of $5 \mathrm{mmol} / \mathrm{l}$ with $[\mathrm{BE}]$ as the criterion, whereas a reduction of only $2 \mathrm{mmol} / \mathrm{l}$ in [BB] indicated the presence of metabolic acidosis by the criterion of [SID]. Therefore, a more sensitive threshold for the diagnosis of metabolic acidosis by means of [SID] might completely explain these results.

The authors presented two cases to exemplify the diagnosis of metabolic acidosis through the Stewart approach, but unfortunately those cases were misinterpreted because the authors incorrectly chose patients with respiratory alkalosis instead of metabolic acidosis. Low [SID] could be a result of the characteristic renal compensation to a respiratory alkalosis, as diagnosed by high $\mathrm{pH}$ and low $\mathrm{PCO}_{2}$ values. As we recently showed, the Stewart approach, used without consideration of the metabolic response to primary respiratory disorders, leads to incorrect diagnoses in $15 \%$ of the cases [2]. The cases shown in the authors' publication illustrate the drawbacks of the Stewart approach in that it likewise led to a misdiagnosis, so that these cases do not exemplify its advantages, but its disadvantages. In addition, we believe the statement that "acidosis is mitigated by hypocapnia" is incorrect. Compensatory responses never overcorrect the $\mathrm{pH}$.

We also regret that the authors failed to look for correlation and agreement between [BE] and [SID]. We showed that these variables are strongly correlated and have narrow limits of agreement [2].

Gunnerson and Kellum considered that $[\mathrm{BE}]$ and $[\mathrm{SID}]$ yield virtually identical results, despite a different conceptual approach to the underlying mechanisms involving acid-base disorders [3]. [SID] might actually be equal to $[\mathrm{BB}]$, the latter having been described over half a century ago [4] $[\mathrm{BB}]$ defines the sum of $\left[\mathrm{HCO}_{3}^{-}\right]$ plus the nonvolatile weak-acid buffers. Both the Stewart and the [BE] methods are general physicochemical approaches that respectively use charge balance and proton balance to express proton concentration. $[\mathrm{BE}]$ is essentially the change in buffer base in vivo and thus shows the change in [SID] from the point where $\mathrm{pH}=7.40$ and $\mathrm{PCO}_{2}=40 \mathrm{mmHg}$ [5].

As we recently reported in a large series of critically ill patients, the use of $\left[\mathrm{HCO}_{3}{ }^{-}\right],[\mathrm{BE}]$ and albumincorrected anion gap has the same diagnostic and prognostic performance as the Stewart approach [2]. The key point is to use those conventional tools properly.

\section{References}

1. Boniatti MM, Cardoso PR, Castilho RK, Vieira SR (2009) Acid-base disorders evaluation in critically ill patients: we can improve our diagnostic ability. Intensive Care Med 35:1377-1382

2. Dubin A, Menises MM, Masevicius FD, Moseinco MC, Kutscherauer DO, Ventrice E, Laffaire E, Estenssoro E (2007) Comparison of three different methods of evaluation of metabolic acid-base disorders. Crit Care Med 35:1264-1270

3. Gunnerson KJ, Kellum JA (2003) Acidbase and electrolyte analysis in critically ill patients: are we ready for the new millennium? Curr Opin Crit Care 9:468-473

4. Singer RB, Hastings AB (1948) An improved clinical method for the estimation of disturbances of the acidbase balance of human blood. Medicine (Baltimore) 27:223-242

5. Kellum JA (2000) Determinants of blood $\mathrm{pH}$ in health and disease. Crit Care 4:6-14

\section{A. Dubin}

Cátedra de Farmacología Aplicada, Facultad de Ciencias Médicas, Universidad Nacional de La Plata, Calle 42 no. 577, La Plata, Buenos Aires 1900, Argentina

A. Dubin $(\bowtie) \cdot$ F. D. Masevicius Servicio de Terapia Intensiva, Sanatorio Otamendi y Miroli, Buenos Aires, Argentina e-mail: arnaldodubin@speedy.com.ar 